\title{
Traumatic paediatric neurosurgical emergencies during the COVID-19 pandemic: experience in a single regional paediatric major trauma centre
}

\author{
Amin Andalib $^{1} \cdot$ Matthew I Sanders $^{1} \cdot$ Saurabh Sinha ${ }^{1}$ \\ Received: 28 August 2020 / Accepted: 22 September 2020 / Published online: 7 October 2020 \\ (C) Springer-Verlag GmbH Germany, part of Springer Nature 2020
}

Dear Editor:

The novel coronavirus (COVID-19), first described in December 2019 in the Wuhan province of China, has created an unprecedented global health crisis. The World Health Organization (WHO) has declared this a pandemic and a global public health emergency. Countries around the globe have come forward with a variety of public health measures, including prohibiting social gatherings as well as closing educational institutions and public places. Moreover, the introduction of widespread "lockdowns" has been seen internationally, with bans on nonessential travel and encouraging new forms of routine, including working from home. The UK government implemented its own nationwide lockdown on 23 March 2020.

Since the beginning of the UK lockdown, there has been increasing concern about an upsurge in non-accidental injuries (NAI) in children [1, 2].

We read with interest the work by Dyson et al. on the incidence of children referred to their institution with suspected NAI during the initial period of lockdown in late March to June of this year [3]. As a regional paediatric major trauma centre (MTC), we questioned whether the published experience of colleagues in a non-MTC was different to our own.

We present a retrospective observational study of all emergency neurosurgical admissions to Sheffield Children's Hospital, a regional paediatric MTC, during the period of lockdown. We also collected retrospective data of a matched control period from the previous year for comparison.

Data were included from all admissions under neurosurgery at Sheffield Children's Hospital from the start of the UK lockdown on 23 March 2020 until 30 June 2020 (“during

Amin Andalib

amin.andalib@nhs.net

1 Department of Neurosurgery, Sheffield Children's Hospital, Sheffield, UK
COVID-19 group"). Corresponding data were retrospectively collected from the same period in 2019 for comparison ("preCOVID-19 group").

An unpaired $t$ test was used to compare mean age between each group. The chi-squared test was used to compare admissions between groups, specifically the number of patients admitted with trauma, as well as NAI as a proportion of total admissions (both traumatic and non-traumatic). Data were analysed using Microsoft Excel.

The total number of patients admitted to neurosurgery both pre- and during COVID-19 were similar across each period, with 34 (17M:17F) and 33 (20M:13F) patients admitted, respectively. Mean $( \pm \mathrm{SD})$ ages of patients in the pre-COVID19 and during COVID-19 groups were $5.41( \pm 3.48)$ and 4.72 $( \pm 3.76)$ years, respectively. There was no statistical difference between gender $(p=0.39)$ or age ( $p=0.44)$.

Breakdown of admissions are summarised in Table 1. There was an increase in patients admitted with trauma during COVID-19 when compared with the pre-COVID-19 group (13 and eight, respectively), which did not reach statistical significance $(p=0.14)$. As a proportion of admissions, patients admitted with trauma almost doubled during COVID$19(57.6 \%$ versus $23.5 \%)$. There was a near fourfold increase in admissions for suspected NAI in the during COVID-19 group when compared with the pre-COVID-19 group (seven and two admissions, respectively), which did not reach statistical significance $(p=0.07)$.

Table 1 Summary of reasons for admission

\begin{tabular}{lll}
\hline & Pre-COVID-19 & During COVID-19 \\
\hline Total admissions & 34 & 33 \\
Non-traumatic & $26(76.5 \%)$ & $19(39.4 \%)$ \\
Traumatic & $8(23.5 \%)$ & $13(57.6 \%)$ \\
Suspected NAI & $2(5.8 \%)$ & $7(21.2 \%)$ \\
\hline
\end{tabular}

NAI, non-accidental injury 
This data shows that there was a definite increase in trauma and NAI as a proportion of total admissions to our centre. The results, however, do not reach statistical significance. This nonsignificant increase reflects the findings of colleagues in paediatric neurosurgical centres elsewhere in the UK [3].

The authors suggest that the lack of significance of results to date may be a consequence of the relatively low numbers of admissions to neurosurgical services, resulting in analyses that are essentially underpowered. This is of grave concern, as statistically significant increased rates of NAI will have a serious impact on child welfare and safety. It is accepted that the likelihood of future lockdowns is high, which has already been demonstrated at a local level and the possibility of further national lockdowns remains substantial.

We propose a national collaboration between paediatric neurosurgical centres in the UK to accurately define the rates of NAI during lockdown periods, which will act to safeguard children and advise legislature in future lockdowns.

In summary, there was a nonsignificant increase in the rates of admission for suspected NAI and trauma to our paediatric neurosurgical major trauma centre during the national lockdown for COVID-19. Further work must be carried out at a national level to better understand the frequency of suspected NAI during periods of lockdown.

Code availability Not applicable.

Authors' contributions AA collected the data. MIS wrote the manuscript. All authors reviewed the manuscript.

Data availability Anonymised data supporting the findings of this work are available upon reasonable request from the corresponding author.

\section{Compliance with ethical standards}

Conflicts of interest None.

Ethics approval The project was registered with and approved by the Sheffield Children's Hospital clinical audit department.

Consent to participate Not applicable.

Consent for publication Not applicable.

\section{References}

1. Baird E (2020). Non-accidental injury in children in the time of COVID-19 pandemic. British Orthopaedic Association. (Published Online $8^{\text {th }}$ April 2020) (https://www.boa.ac.uk/policy-engagement/ journal-of-trauma-orthopaedics/journal-of-trauma-orthopaedicsand-coronavirus/non-accidental-injury-in-children-in-the-time-of. html) [Accessed 19 $9^{\text {th }}$ August 2020]

2. Jay N, Bishop N (2020). What impact is COVID-19 having on child healthcare services? Royal College of Paediatrics and Child Health. (Published Online 15 ${ }^{\text {th }}$ April 2020) (https://www.rcpch.ac.uk/newsevents/news/what-impact-covid-19-having-child-health-services) [Accessed 19 $9^{\text {th }}$ August 2020]

3. Dyson E, Craven C, Tisdall M, James G (2020) The impact of social distancing on pediatric neurosurgical emergency referrals during the COVID-19 pandemic: a prospective observational cohort study. Childs Nerv Syst 3:1-3

Publisher's note Springer Nature remains neutral with regard to jurisdictional claims in published maps and institutional affiliations. 